\title{
Short- and long-term harm resulting from inappropriate antibiotic prescription in childhood and adolescence
}

Over the past decades, there has been an increase in the unjustified prescription of antibiotics for neonates, infants, and children. Until a few years ago, a characteristic of pediatricians was their judiciousness in prescribing antibiotics, thus differentiating themselves from other medical specialties. In general, antibiotics were only used for patients with clinical signs compatible with bacterial infections, and sometimes in indefinite cases. Unfortunately, such adequate strategy has been fading away, and the unjustified use of antibiotics in children has grown exponentially. As I pointed out in an editorial comment ${ }^{1}$ published two years ago, in the United States, the inadequate use of antibiotics has increased in an alarming proportion. Data from the Centers for Disease Control and Prevention (CDC) show that 4 out of 10 children are prescribed antibiotics when seeking care for a common cold, a truly unacceptable approach. It has been estimated that approximately 12 million antibiotic prescriptions are written every year for upper respiratory infections in children, which are obviously not an indication for antibiotic treatment. ${ }^{1}$

What do pediatricians think of this approach? According to a CDC survey, pediatricians point out a lack of time during office visits, pressure from parents, and fear of malpractice liability should the condition be severe. They admit that "prescribing an antibiotic is faster than taking time to discuss, give advice and explain why antibiotics are not good for viral infections".

Antibiotic-related adverse events are very well-known, mainly in newborn infants (NBIs), and they are even more frequent among small preterm infants. In the perinatal period, the prescription of antibiotics to the mother during labor and in the first days of the newborn infant's life lead to major intestinal flora disorders that may affect the physiological microbiota, which plays an essential role in multiple aspects., ${ }^{2,3}$ In a recent study, early antibiotic use was assessed in relation to the benefits of extended breastfeeding. Significantly, infants who received antibiotics showed fewer beneficial effects from breastfeeding. In a subset analysis of fecal microbiota, a significant reduction of Bifidobacterium was observed among children with extended breastfeeding (8-16 months) and early antibiotic use. These results suggest that the long-term metabolic benefits of breastfeeding are conveyed by the intestinal microbiota. ${ }^{4}$

In addition, some studies found that antibiotic use for more than 48-72 hours results in a greater damage, and will increase the risk of necrotizing enterocolitis (NEC), late-onset sepsis, and retinopathy grade 3 among very low birth weight preterm infants. These outcomes may be severe and result in death and future complications. ${ }^{5}$ At present, in most neonatal intensive care units (NICU), more than $80 \%$ of preterm infants are given early antibiotics. ${ }^{5} \mathrm{~A}$ similar situation is observed among NBIs who have fever without a source of infection in their first month of life: they are given antibiotics even though published studies indicate that, in most cases (85\%), fever is caused by a viral infection. Most likely, this approach is affected by fever phobia, which some physicians may have without considering that fever is an important defense mechanism to fight infections.

There is also evidence that the inappropriate use of antibiotics among neonates, especially broad-spectrum agents, increases bacterial resistance, a severe problem. In a study conducted in Australia, it was observed that, in several NICUs, resistance increased even if antibiotics were discontinued at 72 hours. Alarmingly, bacterial resistance in Gram-negative bacilli increased over 25 years at a rate of $2.9 \%$ by year. ${ }^{6}$

Such shocking situation might contribute to the severe consequences of bacterial resistance worldwide, which has therefore resulted in the current enhanced strategies for its prevention.

Infants and young children also develop antibiotic-related disorders (diarrhea, vomiting, bacterial superinfection, etc.). This is more common when broad-spectrum antibiotics are used. For example, amoxicillin/clavulanic acid is commonly used without considering its adverse events.

In addition to the above mentioned adverse events caused by antibiotics in the short term (it is likely that there are more than those mentioned here), in the past years the presence of long-term adverse events has been more accurately defined. This is a highly relevant situation because the negative impact on older children and adults significantly increases the number of affected patients. Unlike short-term effects, those that 
appear in later years may last throughout life.

For several years, it has been known that symptom exacerbation in adults with inflammatory bowel disease (IBD) may be due to antibiotics, although it has also been recently observed in children and, especially, in adolescents. ${ }^{7}$ In 2012, Kronman et al., ${ }^{8}$ published a very interesting study to find out whether exposure of children to antibiotics effective against anaerobic bacteria showed an association with IBD. Out of more than 1000000 patients, 748 developed IBD. The group that had received antibiotics for the past 15 years showed a significant increase in the development of IBD. Exposure to antibiotics before 1 year old has been related to a greater adjusted risk ratio for IBD. The antibiotic most commonly related to IBD was metronidazole, followed by ciprofloxacin.

A recent study included a meta-analysis to assess the risk ratio for gastrointestinal disorders resulting from antibiotic use in children, adolescents and adults. A significant association was observed between antibiotic use and Crohn's disease and IBD. All antibiotics (except penicillin) were associated with the presence of both conditions. Again, the strongest association with Crohn's disease and IBD (OR: 5.01) was observed with exposure to metronidazole, followed by quinolones. $^{9}$

As a corollary of what has been described here about the harmful effects of unjustified antibiotic use, it is necessary to reflect on the serious problems it causes. In everyday practice, the wrongful indication of antibiotics when it is not necessary has become commonplace. It is therefore critical to consider, in every situation that calls for antibiotic prescription, any potential damage that may result from it. It is absolutely necessary that we adopt a different approach and exercise more caution in our practice, taking into consideration that this is an increasingly concerning situation and that only this way we will be able to prevent adverse events resulting from injudicious use of antibiotics, in the shortas well as in the long-term.

José M. Ceriani Cernadas, M.D. Editor-in-chief

http:/ / dx.doi.org/10.5546/ aap.2017.eng.306

To cite: Ceriani Cernadas JM. Short- and long-term harm resulting from inappropriate antibiotic prescription in childhood and adolescence. Arch Argent Pediatr 2017;115(4):306-307.

\section{REFERENCES}

1. CerianiCernadasJM.Laindicación inadecuadaeinnecesaria de antibióticos; un problema creciente. Arch Argent Pediatr 2015;113(1):2-3.

2. Diaz Heijtz R. Fetal, neonatal, and infant microbiome: Perturbations and subsequent effects on brain. Semin Fetal Neonatal Med 2016;21(6):410-7.

3. Hill CJ, Lynch DB, Murphy K, et al. Evolution of gut microbiota composition from birth to 24 weeks in the INFANTMET Cohort. Microbiome 2017;5(1):4.

4. Korpela K, Salonen A, Virta LJ, etal. Association of Early-Life Antibiotic Use and Protective Effects of Breastfeeding Role of the Intestinal Microbiota. JAMA Pediatr 2016;170(8):750-7.

5. Ting JY, Synnes A, Roberts A, et al. Association Between Antibiotic Use and Neonatal Mortality and Morbidities in Very Low-Birth-Weight Infants Without CultureProven Sepsis or Necrotizing Enterocolitis. JAMA Pediatr 2016;170(12):1181-7.

6. CarrD, Barnes EH, Gordon A, et al. Effect of antibiotic use on antimicrobial antibiotic resistance and late-onset neonatal infections over 25 years in an Australian tertiary neonatal unit. Arch Dis Child Fetal Neonatal Ed 2017;102(3):F244-50.

7. Kronman MP, Gerber JS, Prasad PA, et al. Variation in Antibiotic Use for Children Hospitalized With Inflammatory Bowel Disease Exacerbation: A Multicenter Validation Study. J Pediatric Infect Dis Soc 2012;1(4):306-13.

8. Kronman MP,Zaoutis TE, HaynesK, etal. Antibioticexposure and IBD development among children: a population-based cohort study. Pediatrics 2012;130(4):e794-803.

9. Ungaro R, Bernstein CN, Gearry R, et al. Antibiotics associated with increased risk of new-onset Crohn's disease but not ulcerative colitis: a meta-analysis. Am J Gastroenterol 2014;109(11):1728-38. 